\title{
Joint Discussion 4 UV astronomy: stars from birth to death
}

\author{
Ana I. Gómez de Castro ${ }^{1}$ and Martin A. Barstow ${ }^{2}$ (eds.) \\ ${ }^{1}$ Instituto de Astronomía y Geodesia (CSIC-UCM), Facultad de Matemáticas, \\ Universidad Complutense de Madrid, Madrid, Spain \\ email: aig@mat.ucm.es \\ ${ }^{2}$ Department of Physics and Astronomy, University of Leicester, \\ University Road, Leicester LE1 7RH, UK \\ email: mab@star.le.ac.uk
}

\begin{abstract}
The scientific program is presented as well a the abstracts of the contributions. An extended account is published in "The Ultraviolet Universe: stars from birth to death" (Ed. Gómez de Castro) published by the Editorial Complutense de Madrid (UCM), that can be accessed by electronic format through the website of the Network for UV Astronomy (www.ucm.es/info/nuva).

There are five telescopes currently in orbit that have a UV capability of some description. At the moment, only FUSE provides any medium- to high-resolution spectroscopic capability. GALEX, the XMM UV-Optical Telescope (UVOT) and the Swift. Uvot mainly delivers broad-band imaging, but with some low-resolution spectroscopy using grisms. The primary UV spectroscopic capability of HST was lost when the space Telescope Imaging spectrograph failed in 2004, but UV imaging is still available with the HST-wFPC2 and HST-ACS instruments.

With the expected limited lifetime of sl FUSE, UV spectroscopy will be effectively unavailable in the short-term future. Even if a servicing mission of HST does go ahead, to install cos and repair sTIS, the availability of high-resolution spectroscopy well into the next decade will not have been addressed. Therefore, it is important to develop new missions to complement and follow on from the legacy of FUSE and HST, as well as the smaller imaging/low resolution spectroscopy facilities. This contribution presents an outline of the UV projects, some of which are already approved for flight, while others are still at the proposal/study stage of their development.

This contribution outlines the main results from Joint Discussion 04 held during the IAU General Assembly in Prague, August 2006, concerning the rationale behind the needs of the astronomical community, in particular the stellar astrophysics community, for new UV instrumentation. Recent results from UV observations were presented and future science goals were laid out. These goals will lay the framework for future mission planning.
\end{abstract}

Keywords. ultraviolet-general, ultraviolet-solar system, ultraviolet-stars, ultraviolet-ISM, space vehicles-instruments

\section{Preface}

This joint discussion was organized to provide a forum during the IAU General Assembly where the accomplishments of UV astrophysics could be highlighted and a new road map for the future discussed.

The UV range is of prime interest for astrophysics since the resonance lines of the most abundant atoms and ions at temperatures between $3000 \mathrm{~K}$ and $300000 \mathrm{~K}$, together with the electronic transitions of the most abundant molecules $\left(\mathrm{H}_{2}, \mathrm{CO}, \mathrm{OH}, \mathrm{CS}, \mathrm{S}_{2}, \mathrm{CO}_{2}^{+}\right.$, $\left.\mathrm{C}_{2}, \mathrm{O}_{2}, \mathrm{O}_{3}, \ldots\right)$ are at UV wavelengths. After enjoying more than 30 years of continuous access to this range, the astronomical community has been facing uncertain times and provision during the decade 2010-2020 remains so. Coordination is required to define the science goals for the future and the resulting requirements for future UV instrumentation. 
This meeting focused in particular on stellar astrophysics. The understanding of stellar physics is at the very base of our understanding of the Universe. The chemical evolution of the Universe is controlled by stars. Supernovae are prime distance indicators that have allowed to measure the evolution of the curvature of the Universe and to detect the existence of dark energy. The development of life sustaining systems depends strongly on the evolution of stars like our Sun. Some of the most extreme forms of matter in the Universe, the densest and more strongly magnetized, are the magnetars, debris of stellar evolution.

UV instrumentation is required to make progress in these fields. The excellent contributions presented in this Joint Discussion dealt with the many aspects of stellar astrophysics from the analysis of dissipative processes in the atmosphere of cool stars and their impact on the evolution of the planetary systems to the study of the atmospheres and winds of the hot massive stars or the determination of the abundances in white dwarfs. The physics of disks, its role in the evolution of binary systems, and the formation of supernovae were among the main topics treated in the meeting. We should also not forget the role of starbursts and, in general, high-mass stars in the chemical evolution of galaxies. The metallicity gradient in the Galaxy is traced in the UV spectrum of planetary nebulae. The evolution of young planetary disks and the role of the central stars in the photo-evaporation of the giant gaseous planets that have been detected recently. The numerous and high quality contributions to the JD could not fit within the space allowed for these proceedings. However, the full proceedings will be published in extended form in "The Ultraviolet Universe: stars from birth to death" (Ed. Gómez de Castro \& Barstow) published by the Editorial Universidad Complutense de Madrid (UCM) that can be accessed in electronic format through the website of the Network for UV Astronomy (www.ucm.es/info/nuva).

These pages concentrate on reporting on the status of current UV facilities and on the approved and proposed projects for new UV instrumentation. Also, there was an important and interesting discussion about the general needs of the astronomical community that is summarized in the proceedings.

This conference was originally proposed and organized by Willem Wamsteker, who wished to dedicate it to Professor (Emeritus) Cornelius de Jager. Over the years, Professor de Jager contributed significantly to the understanding of stellar atmospheres. Professor de Jager's work on the Sun itself has had a major impact on our understanding of the stellar atmospheres in general. He also played an important role in the IAU through a period when it had to adjust to the new possibilities generated by opening up the space windows.

Sadly, Willem Wamsteker passed away on November 24th 2005, a great loss for all of us. Willem was the director of ESA's IUE Observatory until the mission terminated in 1996. Through his leadership, the IUE data archive became the first fully internet driven astronomical archive. Willem was also a promotor of the NUVA and a key initiator of the World Space Observatory UV (WSO/UV) project; a $1.7 \mathrm{~m}$ UV telescope equipped with state of the art instrumentation providing a factor of 10 improvement on the high resolution spectroscopic capabilities of HST-STIS. The project is driven by a broad international collaboration led by Russia (ROSCOSMOS). His wonderful enthusiasm for science remains with us.

In December 2005, we took over the duty of shaping the scientific program and preparing these proceedings. This would not have been possible without the support of the SOC: T.R. Ayres, W.P. Blair, D. De Martino, M.A. Dopita, J. Grygar, J.B. Hutchings, C. Jordan, Y. Kondo, E.M. Leibowitz, A. Niedzielski, B.M. Shustov, J. Sahade, and Z.R. Wang. 
Members of the NUVA helped with the logistics of the conference organization and the proceedings preparation.

The scientific program is presented as well a the abstracts of the contributions. An extended account of JD04 is published as The Ultraviolet Universe: Stars from Birth to Death, Eds. Ana Ines Gómez de Castro \& Martin A. Barstow (Madrid: Editorial Complutense, ISBN 978-84-7491-852-6) and can be downloaded from the website of the Network for UV Astronomy <www.ucm.es/info/nuva>.

\section{A brief historical introduction to UV astronomy by Cornelis de Jager}

In 1957 Sputnik 1 was launched, followed by a long and ever more sophisticated series of spacecraft. This impressive development in the USSR and USA triggered other nations to follow that example and also to embark in space research. In Europe a preparatory European space research organisation was founded, named COPERS (Commision Prparatoire Europenne pour la Recherche Spatiale). After the preparatory phase the name was changed in ESRO (European Space Research Organisation) and presently the name is ESA.

COPERS established an impressive scientific program that included, among other things, the launching of a $60 \mathrm{~cm}$ UV telescope. Critical questions such as, how Europe with a budget that was less than one tenth of that of NASA, could embark in such an ambitious project, were countered with a reference to the 'Atlantic Factor'. That was kind of a mystical way of reasoning saying that salaries in Europe were considerably less than those in the US and that therefore, Europeans could do much more than NASA with the same amount of money. After a number of years we learned, muddling through, that such is not the case: high-quality labour costs the same. But around 1964 we were not yet at that point. Three competing consortia came into being; each of them made a proposal. These were a French-Belgian-Italian consortium, a German-Dutch one while the British decided to do it all by themselves.

I was involved in the German-Dutch project. It was our first experience in a large international undertaking and I value highly the fine co-operation with our German colleagues. Until a few years ago I kept on a drawer the three voluminous books that resulted from our study.

Evidently the Large European Space-telescope, LAS as it was called, did not come to realisation. The Atlantic Factor appeared not to exist in space research. But the enterprise had its fruits. For Bob Wilson and his British colleagues the study yielded sufficient experience and knowledge to propose to NASA a project that would later become the International Ultraviolet Explorer, IUE. We, in The Netherlands, decided to propose to ESRO the development of a $35 \mathrm{~cm}$ Cassegrain-type UV spectrograph for inclusion in a spacecraft that would later become the TD1A satellite. That spacecraft was a polar orbiter moving in a plane at right angles to the solar direction. An instrument on board of that spacecraft and pointing away from the direction to Earth would thus scan nearly the whole sky in half a year. The telescope could deflect over an angle of some 15 degrees in a direction at right angles to the direction of motion of the spacecraft and over a larger angle in the direction of motion. When a star of sufficient brightness would appear in its field of view the telescope would then automatically point at the star and during some five minutes it would stay so. During that time the UV spectrum should be scanned in three wavelength regions. The data were to be stored on a tape recorder and 
twice a day, when the satellite would pass over the European ground station the data would be collected.

Satellite launching (12 March 1972) went excellent and the first spectra came streaming in; they were fine.

A week or so later, at a conference in Madrid, I met Ernst Trendelenburg, who later would become scientific director of ESRO. "How are you feeling", he called at me. "Fine", I said, "enjoying our wonderful spectra". "Fine???" he wondered, "Don't you know that the tape recorder broke down? No more data." What a misery!

ESRO could partially cure the situation by establishing a number of improvised ground stations over the globe. One of then was on a location as remote as Easter Island. That way, in the course of two yeas we still succeeded in getting spectra of some 200 stars.

In the mean time things had developed. Morton and his colleagues had obtained the first UV rocket spectra of some stars in Orion. These were marvellous. For the first time we got direct evidence of the large rate of mass loss of early type stars. Lyman Spitzer developed his idea for a very large astronomical telescope that would later become the Hubble Space Telescope. Not everyone believed him. In 1969 we organised the first international symposium on UV astronomy, IAU Symposium No. 36. It was in Lunteren, the Netherlands, and among the 150 participants were famous astrophysicists as well as many youngsters like young Rashid Sunyaev and young Roger Bonnet. At the closing dinner there were a number of speakers telling funny stories. Laughter all over the place. Then Spitzer's talk came. People were again expecting a funny story and when he told of his idea for a $2.5 \mathrm{~m}$ telescope in space the attendance became hilarious. "Expected lifetime 10 years". Bursts of laughter again. A youngster asked me "Who is that fellow. He is the most funny of all". I had to explain to him that he was listening to one of the great astronomers of the century and that this 'funny fellow' was deadly serious. Gradually the audience became to realize that they were actually listening to a visionary scientist with far reaching ideas.

About half a year after TD1A (21 August 1972) the Orbiting Astronomical Observatory Copernicus was launched. It was designed to obtain UV spectra in several bands between 100 and $>300 \mathrm{~nm}$.

After the termination of our TD1A experiment we realized that in some three, four, years IUE would be launched and we decided to fill that gap with a balloon experiment, together with US colleagues. The Balloon Ultra-violet Stellar Spectrometer BUSS had a spectral resolution that was slightly better than IUE. It was launched a dozen of times during several launching campaigns in the US.

I am pleased to see in this room several of the participants in the BUSS campaigns. Thank you again for this fine co-operation. The period that I described here was for me really a great time.

There is one person in particular that I am greatly missing. Willem Wamsteker, who has served the community so immensely and thoughtfully, told me last year that this meeting would be dedicated to me. While I felt honoured I told him that there are others who more deserve this honour. I wish that this conference could be dedicated to Willem.

Willem, we will not forget you.

\section{Star formation and young stellar objects}

\subsection{Young Stellar Objects: accretion and outflow}

Ana I. Gómez de Castro: Young stellar objects are an excellent laboratory to study the physics of accretion and outflow. The relevance of ultraviolet astronomy to study the 
physics of these objects is reviewed in this contribution. Special emphasis is made in the following aspects:

(a) The magnetic interaction between the star and the disk produces high energy radiation and particles that heat the inner disk. The spectral output form this region is described and compared with the theoretical predictions.

(b) $\mathrm{H}_{2}$ and $\mathrm{CO}$ electronic transitions are observed in the spectra of the T-Tauri stars allowing an analysis of the circumstellar/disk warm material and its interaction with radiation.

\subsection{High-mass stars: starbursts}

Rosa M. González Delgado: Starbursts are the preferred place where massive stars form; the main source of thermal and mechanical heating in the interstellar medium, and the factory where the heavy elements form. Thus, starbursts play an important role in the origin and evolution of galaxies. Starbursts are bright at ultraviolet (UV) wavelengths, and after the pioneering IUE program, high spatial and spectral resolution UV observations of local starburst galaxies, mainly taken with HST and FUSE, have made relevant contributions to the following issues: $(a)$ the determination of the initial mass function in violent star forming systems in low- and high-metallicity environments, and in dense (e.g., in stellar clusters) and diffuse environments; (b) the modes of star formation: Starburst clusters are an important mode of star formation; $(c)$ the role of starbursts in $\mathrm{AGN} ;(d)$ the interaction between massive stars and the interstellar and intergalactic media; and $(e)$ the contribution of starbursts to the re-ionization of the universe. Despite the very significant progress obtained over the past two decades of UV observations of starbursts, there are important problems that still need to be solved. Highspatial resolution UV observations of nearby starbursts are crucial to further progress in understanding the violent star formation processes in galaxies, the interaction between the stellar clusters and the interstellar medium, and the variation of the IMF. Thus, a new UV mission furnished with an intermediate spectral resolution long-slit spectrograph with high spatial resolution and high UV sensitivity is required to further progress in the study of starburst galaxies and their impact on the evolution of galaxies.

\section{Life in main sequence}

\subsection{Atmospheres and winds in cool stars}

Isabella Pagano: Cool star outer atmospheres represent an important laboratory in which solar-like magnetic activity phenomena can be studied under a wide variety of conditions, allowing us to gain insight into the fundamental processes involved.

The UV range is especially useful for such studies because it contains powerful diagnostics extending from warm $(10000 \mathrm{~K})$ chromospheres out to hot $(1-10 \mathrm{MK})$ coronae. Also very weak coronal winds from cool stars have been identified and characterized thanks to high resolution UV spectra.

Here I give a review on UV spectroscopy main achievements for cool star chromospheres, transition regions, coronae and coronal winds. I also outline the requirements for future experiments able to allow progresses in this field.

\subsection{Evolution over time of magnetic dynamo driven $U V$ emissions of $d G-d M$ stars and effects on hosted planets}

Eduwrd F. Guinan, Ignasi Ribas, and S.G. Engle The evolution over time of the magnetic activity and the resulting X-ray and UV coronal and chromospheric emissions of main-sequence $\mathrm{dG}$, dK, and $\mathrm{dM}$ stars with widely different ages will be discussed. 
Young cool stars spin rapidly and have correspondingly very robust magnetic dynamos and strong coronal and chromospheric X-ray-UV (XUV) emissions. However, these stars spin-down with time as they lose angular momentum via magnetized winds and their magnetic generated activity and emission decrease. For example, the study of solar proxies shows that the young Sun was rotating more than ten times faster than today and had correspondingly very high levels of magnetic activity and very intense X-ray-UV (XUV) emissions. Studies of dK-dM stars over a wide range of ages and rotations show similar (but not identical) behavior. Particular emphasis will be given to discussing the effects that UV emissions have on the atmospheres and evolution of solar system planets as well as the increasing number of exoplanets found hosted by $\mathrm{dG}-\mathrm{dM}$ stars. The results from modeling the early atmospheres of Venus, Earth and Mars using recently determined XUV irradiances and winds of the young Sun are also briefly discussed. For example, the loss of water from juvenile Venus and Mars can be explained by action of the strong XUV emissions and robust winds of the young Sun. We also examine the effects of strong X-ray and UV coronal and chromospheric emissions (and frequent flares) that dM stars may have on possible planets orbiting within their habitable zones (HZ) - located close to the low luminosity host stars $(0.05<\mathrm{HZ}<0.4 \mathrm{AU})$. Dwarf $\mathrm{M}$ stars make interesting targets for further study because of their deep convective zones, efficient dynamos and strong XUV emissions. Furthermore, a large fraction of dM stars are very old (>5 Gyr), which present possibilities for the development of highly advanced modes of intelligent life on planets that may orbit them. This research is supported by grants from NASA and utilizes data from the IUE, FUSE, HST, EUVE, ROSAT, XMM, and the Chandra missions. We are very grateful for this support.

\subsection{Systematics of $O B$ spectra}

Nolan R. Walborn: The systematics of OB spectra are reviewed in the optical domain, dominated by photospheric lines, and in the far ultraviolet (both IUE and FUSE ranges), in which the stellar-wind profiles dominate. First, the two-dimensional (temperature, luminosity) trends in normal spectra are surveyed. Then, the normal reference frame having been established, various categories of peculiar objects can be distinguished relative to it, which reveal several phenomena of structural and/or evolutionary significance. Included are CNO anomalies at both early and late O-type stars, three varieties of rapid rotators, hot and cool Of/WN transition objects, and the recently discovered second known magnetic O-type star. The importance of both optical and UV observations to understand these phenomena is emphasized; for instance, progress in understanding the structure of the new O-type magnetic oblique rotator is hampered by the current lack of a UV spectrograph. While progress in the physical interpretation of these trends and anomalies has been and is being made, increased attention to modeling the systematics would accelerate future progress in this author's opinion. Finally, preliminary results from a Chandra high-resolution survey of OB-type star X-ray spectra (PI: W. Waldron) are presented. They provide evidence that, just as emerged earlier in the UV, systematic morphological trends exist in the X-ray domain that are correlated with the optical spectral types, and hence the fundamental stellar parameters, contrary to prevailing opinion.

\subsection{High mass stars: stellar populations}

Luciana Bianchi: Massive stars dominate the chemical and dynamical evolution of the ISM, and ultimately of their parent galaxy and the universe, because of their fast evolution and intense supersonic winds. Decades ago, the advent of space-born UV spectroscopy marked the discovery of mass loss in massive stars and began to change our understanding of their evolution. Recently, significant advances in stellar modeling, and 
the observation of crucial ions in the far-UV spectral range disclosed by FUSE, led to resolve long-standing issues in modeling massive star winds and atmospheres, and to assess of the roles of shocks and clumping. A revised (downwards) calibration of Teff for early spectral types emerged as a result. Meanwhile, HST imaging had opened the possibilty of detailed resolved studies of stellar populations in Local Group galaxies, sampling a variety of metallicity and environment conditions. More recently, GALEX is providing the first global, deep view of the massive star populations for hundreds of nearby galaxies. The unprecedented coverage and sensitivity of the GALEX UV imaging, easily detecting extremely low levels of SF, is once again revolutionizing some of our views of massive star formation in galaxies.

\section{Star death}

\subsection{Planetary Nebula}

Letizia Stanghellini: The asymptotic giant branch (AGB) phase of stellar evolution is common to most stars of low and intermediate mass. Most of the carbon and nitrogen in the Universe is produced by AGB stars. The final fate of the AGB envelopes are represented by planetary nebulae $(\mathrm{PN})$. By studying $\mathrm{PN}$ abundances and compare them with the yields of stellar evolution is possible to quantify carbon and nitrogen production, and to study cosmic recycling in galactic and Magellanic Cloud populations. In this paper we present the latest results in $\mathrm{PN}$ chemical abundance analysis and their implication to the chemical evolution of the galaxy and the Magellanic Clouds, with particular attention to carbon abundance, available only thanks to ultraviolet spectroscopy.

\subsection{Planetary Nebulae abundances and stellar evolution}

Stuart R. Pottasch: A summary is given of planetary nebulae abundances from ISO measurements. It is shown that these nebulae show abundance gradients (with galactocentric distance), which in the case of neon, argon, sulfur and oxygen (with four exceptions) are the same as HII regions and early type star abundance gradients. The abundance of these elements predicted from these gradients at the distance of the Sun from the center are exactly the solar abundance. Sulfur is the exception to this; the reason for this is discussed. The higher solar neon abundance is confirmed; this is discussed in terms of the results of helioseismology. Evidence is presented for oxygen destruction via $\mathrm{ON}$ cycling having occurred in the progenitors of four planetary nebulae with bilobal structure. These progenitor stars had a high mass, probably greater than $5 \mathrm{M}_{\odot}$. This is deduced from the high values of $\mathrm{He} / \mathrm{H}$ and $\mathrm{N} / \mathrm{H}$ found in these nebulae. Formation of nitrogen, helium and carbon are discussed. The high-mass progenitors which showed oxygen destruction are shown to have probably destroyed carbon as well. This is probably the result of hot bottom burning.

\subsection{Supernovae and GRB progenitors}

Stephen J. Smartt: Over the past five years we have been attempting to directly detect the massive progenitor stars of nearby supernovae $(<20 \mathrm{Mpc})$ in archive $H S T$ images, combined with an HST T-o-O programme in Cycles 10-14. We have detected the progenitors of three type II-P SNe in M 74 and M 51, showing them to be red supergiants with initial masses close to the theoretical limit for core-collapse $\left(8 \mathrm{M}_{\odot}\right)$. We have set robust upper luminosity and mass limits on another 12 progenitor stars (from type II-P, and $\mathrm{Ib} / \mathrm{c}$ ) supernovae. There are three striking results so far from this project. One is that we have observationally confirmed for the first time that the common type II-P $\mathrm{SNe}$ do indeed come from red supergiants. The second is that II-P SNe only appear to 
arise in stars with initial masses less than $15 \mathrm{M}_{\odot}$. Thirdly, we find that faint SNe which have been thought to come from black-hole forming SNe are more likely to have lowmass progenitors. We present the results of this survey and review the work from other competing groups in the field.

\section{Compact Objects}

6.1. The importance of UV observations for the study of white dwarfs and the local interstellar medium

Martin A. Barstow: The development of far-UV astronomy has been particularly important for the study of hot white dwarf stars. A significant fraction of their emergent flux appears in the far-UV and traces of elements heavier than hydrogen or helium are, in general, only detected in this waveband or at shorter wavelengths that are also only accessible from space. Although white dwarfs have been studied in the far-UV throughout the past $\sim 25 \mathrm{yr}$, since the launch of IUE, only a few tens of objects have been studied in great detail and a much larger sample is required to gain a detailed understanding of the evolution of hot white dwarfs and the physical processes that determine their appearance. This talk reviews the current knowledge regarding hot white dwarfs and outlines what work needs to be carried out by future far-UV observatories.

\subsection{UV observations of interacting binaries}

Knox S. Long: Virtually all of the exotic objects in the Galaxy are the products of binary evolution, including binary pulsars, black-hole candidates, low mass X-ray binaries, cataclysmic variables and symbiotic stars. Type Ia supernovae, the standard candle of modern cosmology, are produced by them. UV spectroscopy of interacting binaries obtained with HST and FUSE have dramatically improved our understanding of interacting binaries and of the wide range of physical processes that characterize their emission. UV imaging has made it possible to isolate binaries and the products of binary evolution in old stellar populations and thereby test directly models of binary evolution in dense stellar systems. In this review, I will summarize some of the highlights of what we have learned about binaries and their evolution from UV observations, and suggest how, if suitable instrumentation were to exist, furthur observations could resolve some of the important questions that remain.

\subsection{RS Ophiuchi in outburst, seen with XMM-Newton and Chandra}

M. Orio, R. Gonzalez-Riestra, Elia Leibowitz, T. Nelson, and E. Tepedenlelioglu: We present X-ray observations of the recurrent nova RS Ophiuchi in outburst, obtained with the Chandra and XMM-Newton observatories on six different dates post discovery. An important goal of the observations was to study the central UEV-supersoft X-ray source. X-ray grating spectra are the best way to place constraints on the physical parameters of the white dwarfs in these systems. In the first month after the outburst, we detected emission lines of $\mathrm{H}$ and $\mathrm{He}$-like ions of metals including Si, $\mathrm{Mg}$ and Ne. The presence of these lines indicates a range of plasma temperatures, and most likely we were detecting emission from red giant wind material shocked by the nova ejecta. Later spectra show a large increase in emission at longer wavelengths, peaking two months after the outburst. We attribute this to the emergence of the underlying white dwarf. We used NLTE white dwarf atmosphere models to fit the spectra, finding $\log g=9$, and $T \simeq 800000 \mathrm{~K}$. This indicates a white dwarf mass of $>1.2 \mathrm{M}_{\odot}$. We also present a preliminary timing analysis of the XMM-Newton light curves. We find a period of $\sim 35 \mathrm{~s}$ both in March and April. Although the origin of this period is still a question for 
debate, it may be the spin period of the white dwarf. If this is the case, the white dwarf must again be very massive, making RS Ophiuchi a likely supernova Type Ia progenitor.

\section{The impact of stellar astrophysics on cosmological evolution}

\subsection{Missing baryons: the Local Group and beyond}

F. Nicastro, S. Mathur, M. Elvis, Roy Williams, and F. Fiore: More than half $(\sim 54 \%)$ of the expected number of baryons in the local Universe have eluded detection until very recently, due to their high temperature $\left(T \simeq 10^{5}-10^{7} \mathrm{~K}\right)$ and low density $\left(n_{b} \simeq 10^{-6}-10^{-5} \mathrm{~cm}^{-3}\right)$. At redshift $z>2$ most of the baryons lie in the Intergalactic Medium, in a mildly photo-ionized (by the metagalactic radiation field) phase, and they are easily observed and counted in the branches and trees of the so called Ly-alpha forest. However, at $z<2$, intervening $\mathrm{H}$ I Ly-alpha absorption becomes progressively rarer, while already virialized structures, or structures in the way of forming, account for only about $20-30 \%$ of the baryons. Theory predicts that the majority of baryons get shock-heated during the continuous process of structure formation and so becomes invisible to optical or UV photons at $z \leqslant 2$. Metals in such high-temperature medium, however, would still produce some opacity to soft X-ray and Far-Ultraviolet (FUV) photons, mainly through $\mathrm{K} \alpha$ resonant transitions from Li-like (FUV/X-rays), He-like and H-like (X-rays) ions of $\mathrm{C}, \mathrm{N}, \mathrm{O}$ and $\mathrm{Ne}$.

In this contribution, I review recent observational efforts towards the detections of the so-called missing baryons in the local Universe, both in our own Local Group and at cosmological distances. I will show how both X-ray (Chandra) and FUV (FUSE) data clearly point toward the presence of a large amount of hot, shock-heated, gas $\left(T \simeq 10^{6} \mathrm{~K}\right)$ either forming an extended Galactic halo $(\geqslant 200 \mathrm{kpc})$ or an even more extended $(2-4 \mathrm{Mpc})$ Warm-Hot Intergalact Medium filament of metal-enriched primordial material, connecting our own Local Group possibly with the Virgo Cluster, and in which our Galaxy is embedded. I present the first detections of WHIM filaments at cosmological distance, and show how these first measurements are consistent with both theoretical predictions and the number of missing baryons, within the still large uncertainties due to the low number statistics.

\section{The impact of stellar astrophysics in understanding the formation of life sustainable systems}

\subsection{Young planetary disks}

Alain Lecavelier des Étangs: Young planetary disks have been widely observed in the ultraviolet during the last two decades. The sensitivity to electronic transition of atoms and ions in the near ultraviolet, and of molecules like $\mathrm{CO}$ and $\mathrm{H}_{2}$ in the far ultraviolet, lead to detailed observations of the gaseous content of disks surrounding pre-main sequence and main sequence stars.

The main results are the measurements of the physical characteristics of the molecular disk in proto-planetary systems, and the description of the gas phase in few debris disks.

In the very particular case of the $\beta$ Pictoris disk, UV and optical spectroscopy lead to the extraordinary discovery of extra-solar comets falling onto the star. We are witnessing a very strong cometary activity in a young planetary system, likely similar to our own Solar system, four billion years ago.

\subsection{Extrasolar planets atmospheres}

Alfred Vidal-Madjar: Almost 200 extrasolar planets have been discovered since 1995, when the first one was detected orbiting the solar type star $51 \mathrm{Peg}$. The extrasolar planets 
known so far show a variety of characteristics, in some cases not at all represented in our solar system. The "hot Jupiters", in particular, were not predicted by models before being discovered: they are gas giant planets orbiting at only few stellar radii. This peculiarity gives us the unique opportunity of exploring their upper atmosphere while they transit in front of their parent star. Among the atmospheric species detected so far with this method, we can list NaI, in the visible, and HI, O I and C II in the far UV. These UV detections, in particular, unveiled a even more striking feature: the upper atmosphere of these exoplanets is evaporating at a very fast pace, in a "blow off" process.

\section{Poster contributions}

\subsection{AGB Stars: testing carbon loss via the ultraviolet lines}

Yulia V. Milanova, and Alexander F. Kholtygin: A method is proposed to determine the realistic abundances of carbon in planetary nebulae based on the actual distribution functions of errors in measuring line intensities. Fluctuations both in temperature and in mass density in a nebula are taken into account. The $\mathrm{C}$ abundances and the amplitudes of temperature and density fluctuations for the large sample of $\mathrm{PNe}$ are given. The intensity of the ultraviolet lines of $\mathrm{C}$ ions are used for determining the more exact abundances. These abundances are probably the most reliable at the present time and give estimations of the primordial CNO abundances at the epoch when the progenitors of PNs are formed. Based on the newly carbon abundances, the total mass losses of carbon during the AGB stage of evolution are estimated.

\subsection{On the origin of two-shell supernova remnants}

Vasilii V. Gvaramadze: It is known that proper motion of massive stars causes them to explode far from the geometric centers of their wind-driven bubbles and thereby affects the symmetry of the resulting diffuse supernova remnants (SNRs). We use this fact to explain the origin of SNRs consisting of two partially overlapping shells (e.g., 3C 400.2, Cygnus Loop, Kes 32, etc.), whose unusual morphology is usually treated in terms of the collision (or superposition) of two separate SNRs or breakout phenomena in a region with a density discontinuity. We propose that a SNR of this type is a natural consequence of an off-centered cavity supernova (SN) explosion of a moving massive star, which ended its evolution near the edge of the main-sequence (MS) wind-driven bubble. Our proposal implies that one of the shells is the former MS bubble reenergized by the SN blast wave. The second shell, however, could originate in two somewhat different ways, depending on the initial mass of the SN progenitor star. It could be a shell swept-up by the SN blast wave expanding through the unperturbed ambient interstellar medium if the massive star ends its evolution as a red supergiant (RSG). Or it could be the remainder of a preexisting shell (adjacent to the MS bubble) swept-up by the fast progenitor's wind during the late evolutionary phases if after the RGS phase the star evolves through the WolfRayet (WR) phase. In both cases the resulting (two-shell) SNR should be associated only with one (young) neutron star (thus one can somewhat improve the statistics of neutron star/SNR associations since the two-shell SNRs are quite numerous). We discuss several criteria to discern the SNRs formed by SN explosion after the RGS or WR phase.

\subsection{Analysis of the high-temperature region in Be-type stars}

Andrea F. Torres, Adela E. Ringuelet: The High Temperature Region (HTR) that surrounds the photospheres of Be-type stars is studied in order to derive observational constraints for modelling Be-type stars, in particular for the region where superionization takes place. 50 Be-type stars, representative of a considerable range of temperature, were 
chosen. From archival, high-dispersion IUE spectra, different lines that originate in the HTR region were considered, namely the resonance lines of Si IV, C IV and AlIII, and He II 1640. Equivalent widths (corrected for photospheric contribution), optical depths, atom columns and expansion velocities were measured. From this observational data several correlations between different observables were obtained. These correlations permit us to discuss the geometry, density distribution and heat input of the lines formation regions (LFRs). The major results can be summarised as follows:

(a) The circumstellar material contributes to the resonance lines of Si IV, C IV, Al III and to the He II 1640 at all inclination angles.

(b) In Si IV, C IV and Al III the equivalent widths have a tendency to increase in objects with high rotational velocities.

(c) Si IV and C IV equivalent widths are also correlated to the kinetic energy of the expansion velocity. This means that dissipation of mechanical energy is one of the heating mechanisms.

(d) On the basis of the expansion velocities and the line profiles, we establish a sequence for the LFRs: The LFR of He II is at the base of the wind and the closest to the central star. The LFRs of Si IV and C IV are inmersed in the stellar wind. The LFR of Al III is an interface between the HTR and the cool envelope.

The analysis followed in this work is completely model-independent. Consequently, these results could be useful to decide which are the facts that are to be considered when modelling Be-type stars.

\subsection{High resolution spectroscopy of halo stars in groundbased $U V$}

Valentina Klochkova, Gang Zhao, S. Ermakov, and Vladimir Panchuk: For the first time an atlas of high-spectral resolution $(R=60000)$ CCD-spectra in the low studied wavelength range $3500-5000 \AA$ is presented for four stars with values of metallicity $-3.0<[\mathrm{Fe} / \mathrm{H}]<-0.6$, temperatures $4750<T_{\mathrm{e}}<5900 \mathrm{~K}$, and surface gravity $1.6<\log g$ $<5$.0. Based on these spectral data we determined model atmosphere parameters and calculated abundances of 29 chemical elements or their ions.

9.5. Hyper ionization phenomena in the $C \mathrm{IV}, N \mathrm{IV}$, and $N \mathrm{~V}$ regions of 20 Oe-type stars, including HD 93521

Antonios Antoniou, E. Danezis, Evaggelia Lyratzi, D. Nikolaidis, L.C. Popovic, and M.S. Dimitrijevic: As it is already known, the spectra of many Oe- and Be-type stars present Discrete Absorption Components (DACs) which, due to their profiles' width as well as the values of the radial velocities, create a complicated profile of the main spectral lines. In this poster paper we detect the presence of this phenomenon (DACs or SACs) in the C IV resonance lines, the N IV spectral line, and the N V resonance lines of 20 Oe-type stars of different spectral subtypes. In particular we discuss these lines in the spectrum of the star HD 93521 which is a relatively bright, very rapidly rotating $\mathrm{O} 9.5 \mathrm{~V}$ star.

Method: In our study we apply the method proposed by Danezis et al. on the IUE spectra of 20 Oe-type stars, including the star HD 93521 observed with IUE from 1979 until 1995, and we examine the time variations of the physical parameters, stated below, as a function of the spectral subtype.

Results: As a first result we detect that the C IV resonance lines, the N IV spectral line, and the $\mathrm{N} v$ resonance lines each consist of one to five Satellite Absorption Components (SACs or DACs). With the above method we calculate the values of the apparent rotational and radial velocities, the Gaussian standard deviation of the random motions of the ions, the random velocities of these motions, as well as the optical depth, the column 
density, the Full Width at Half Maximum (FWHM), the absorbed and the emitted energy of the independent regions of matter which produce the main and the satellites components of the studied spectral lines.

Discussion: We point out that the new and important aspect of our study is the values' calculation of the above parameters, their time scale variations and their variations as a function of spectral subtype,using the DACs or SACs theory. Our results are a successful test of this theory and of Danezis et al. proposed method. This study is a part of a Ph.D. Thesis.

\subsection{Study of Ho regions in 120 Be-type stars, and the complex structure of the Si IV 1393.755, 1402.77 A regions of 68 Be-type stars}

Evaggelia Lyratzi, E. Danezis, Antonios Antoniou, D. Nikolaidis, L.C. Popovic, and M.S. Dimitrijevic: As it is already known, the spectra of many Oe- and Be-type stars present Discrete Absorption Components (DACs) which, due to their profiles' width as well as the values of the radial velocities, create a complicated profile of the main spectral lines. In this poster paper we detect the presence of this phenomenon (DACs or SACs) in the shape of $\mathrm{H} \alpha$ line in the spectra of 120 Be-type stars, and in the Si IV resonance lines in the spectra of 68 Be-type stars of all the spectral subtypes and luminosity classes.

Method: In our study we apply the method proposed by Danezis et al. on the stellar spectrographs of 120 Be-type stars which were taken by Fehrenbach and Andrillat (resolution 5,5 and $27 \AA$ with the telescope of $152 \mathrm{~cm}$ in the Observatory of Haute Provence), and on the spectra of 68 Be stars observed with IUE, and we examine the variations of the physical parameters, stated below, as a function of spectral subtype and luminosity class.

Results: We find that in the Be-type stellar atmospheres, there are two regions that can produce the $\mathrm{H} \alpha$ Satellite Absorption Components (SACs or DACs). The first one lies in the chromosphere and the second one in the cool extended envelope. With the above method we calculate: (a) For the chromospheric absorption components we calculated the optical depth as well as the rotational and radial velocities of the independent regions of matter which produce the main and the satellites components. $b$ ) For the emission and absorption components which are created in the cool extended envelope we calculated the FWHM, the optical depth and the radial velocities of the independent regions of matter which produce the main and the satellites components.

We find that the absorption atmospherical regions where the Si IV resonance lines originated may be formed of one to five independent density layers of matter which rotate with different velocities, producing one to five Satellite Absorption Components (SACs or DACs). With the above method we calculate the values of the apparent rotational and radial velocities, as well as the optical depth of the independent regions of matter which produce the main and the satellites components of the studied spectral lines.

Discussion: We point out that the new and important aspect of our study is the values' calculation of the above parameters and their variations as a function of spectral subtype and luminosity class, using the DACs or SACs theory. Our results are a successful test of this theory and of Danezis et al. $(2003,2005)$ proposed method. This study is a part of a Ph.D. Thesis.

\subsection{A new approach for DACs and SACs phenomena in the atmospheres of hot emission-line stars}

D. Nikolaidis, E. Danezis, Evaggelia Lyratzi, L.C. Popovic, M.S. Dimitrijevic, Antonios Antoniou, and E. Theodossiou: As it is already known, the spectra of 
many Oe- and Be-type stars present Discrete Absorption Components (DACs) which, due to their profiles' width as well as the values of the radial velocities, create a complicated profile of the main spectral lines. This fact is interpreted by the existence of two or more independent layers of matter, in the region where the spectral lines are formed. Such a structure is responsible for the formation of a series of satellite components (DACs or SACs) for each spectral line (Bates \& Halliwell, 1986, Danezis et al. 2003, 2005).

Method: In this paper we present a mathematical model reproducing the complex profile of the spectral lines of Oe-type and Be-type stars that present DACs or SACs. This model presupposes that the regions, where these spectral lines are formed, are not continuous but consist of a number of independent absorbing or emitting density layers of matter and an external general absorption region. In this model we assume that the line broadening is due to the random motion of the ions and the rotation of the density regions that produce the spectral line and its satellite components. With this method we can calculate the values of the apparent rotational and radial velocities, the Gaussian standard deviation of the random motions of the ions, the random velocities of these motions, as well as the optical depth, the Full Width at Half Maximum (FWHM), the absorbed and the emitted energy and finally the column density of the independent regions of matter which produce the main and the satellites components of the studied spectral lines.

Results: In order to check the above spectral line function, we calculated the rotational velocity of HeI $4387.928 \AA$ absorption line in the spectra of five Be-type stars, using two methods, the classical Fourier analysis and our model. The values of the rotational velocities, calculated with Fourier analysis, are the same with the values calculated with our method.

Discussion: We point out that the new and important aspect of this method is the values' calculation of the above parameters using the DACs or SACs theory.

\subsection{Eta Carinae: what we have learned from HST-STIS in the UV}

Theodore R. Gull: The Luminous Blue Variable $\eta$ Carinae is revealing many answers to its mysteries by high spatial resolution in the visible and the ultraviolet. Studies with the HST-STIS from 1998.0 to 2004.3 show major changes in the stellar and nebular spectra that track with the 2024-day period first noted by A. Damineli in the visible and followed by M. Corcoran via RXTE x-ray monitoring. We will show examples of the stellar and nebular spectra indicating changes in the central source, likely a massive binary system and indicating the response of the nebular ejecta, which is the $>12 \mathrm{M}_{\odot}$ Homunculus, the $0.5 \mathrm{M}_{\odot}$ Little Homunculus, both bipolar structures, with intervening skirts. Within the interior skirt are located the Weigelt blobs, B, C and D, plus the Strontium Filament, all of which respond to the strong UV emission originating from the hot, less massive companion. Narrow-line absorption systems correlate with the Homunculus and Little Homunculus and are seen in hundreds of metal lines. For the Homunculus, the metal energy level populations correspond to $760 \mathrm{~K}$, but the $\mathrm{OH}, \mathrm{CH}, \mathrm{NH}$ and $\mathrm{CH}+$ to $60 \mathrm{~K}$, while nearly a thousand $\mathrm{H}_{2}$ lines are visible during the broad maximum. The Little Homunculus has a kinetic temperature of $\sim 6400 \mathrm{~K}$ during the broad maximum, but drops to $5000 \mathrm{~K}$ during the short minimum. Much is being learned about the N-rich, C,Opoor chemistry of this ejecta from a massive star in the late stages of CNO-processing. Recent GRB spectra show similar hot metal absorption gases likely being the ejecta from progenitor stars. Were they Wolf-Rayet stars?

9.9. High resolution echelle spectrograph NES for visible and groundbased UV regions

Vladimir E. Panchuk, Valentina G. Klochkova, I.D. Najdenov, and Maxim V. Yushkin: We present the high-resolution echelle spectrograph NES of the $6 \mathrm{~m}$ telescope. 
The NES is located at the Nasmyth focus (1:30) platform of the telescope. The NES in combination with the image slicer provides a spectral resolution of $R=75000$ within the spectral region 3200 - $10000 \AA$.

\subsection{Absorption profiles of Lyman- $\gamma$ of atomic hydrogen perturbed by collisions with protons}

Nicole F. Allard, and I. Noselidze: A strong feature near $995 \AA$ in the Lyman- $\gamma$ wing of hot white dwarfs has been demonstrated to be due to quasi-molecular absorption of $\mathrm{H}_{2}$ molecule. We present new theoretical calculations of the line profile of Lyman gamma that include the variation of the radiative dipole moment during the collision.

\subsection{Activity of supersoft X-ray sources: the case of V Sge}

Vojtech Simon, and Janet A. Mattei: Super-soft X-ray sources (SSXSs) are unique binary systems with a very high mass transfer rate onto the white dwarf (WD) (dm/dt $\left.\simeq 10^{-} 7 \mathrm{M}_{\odot} / \mathrm{yr}\right)$. Such a high $\mathrm{dm} / \mathrm{dt}$ gives rise to a steady nuclear burning of accreted hydrogen on the surface of the WD (e.g., the model by van den Heuvel et al. 1992), and consequently to an intense soft X-ray emission. Its reprocessing on the disk and donor makes SSXS bright source in the ultraviolet and optical. Most SSXS are observed in the Magellanic Clouds. V Sge is one of a few SSXSs located in our Galaxy and is called the representative of the V Sge-type class. This system is very active in various spectral passbands. Here we concentrate on the long-term optical activity of this system in the context of SSXSs. We show that this activity in V Sge underwent dramatic evolution during the recent decades. We also find that in so-called active segments (usually displaying high/low states) cycles are often apparent but their length undergoes large, often gradual variations. We give the implications for the accretion wind evolution (model by Hachisu and Kato 2003): an increase of the mass outflow rate from the donor is in plausible agreement with the observations. We also analyse the color indices and absolute magnitudes of V Sge and compare them with those of the 'classical' (SSXBs), the V Sge-type class, the symbiotic SSXBs, and the classical novae in the soft X-ray phase. This approach helps comparing the properties and configuration of the reprocessing medium in the individual systems. We also show that there is no systematic difference between the classical SSXBs and the VSge-type class, as regards their $V$ - and $U$-band absolute magnitudes. Since soft X-rays are easily absorbed, only a small fraction of SSXSs is identified as such. The activity and properties in the ultraviolet and optical can thus serve as a useful method for identification of SSXSs.

\subsection{Distance to Wolf-Rayet Star WR 134}

Krzysztof Czart, and Andrzej Strobel: Distances to Wolf-Rayet stars are still the subject of research. The only WR star with reliable Hipparcos parallax is WR 11 (van der Hucht et al. 1997). Other Wolf-Rayet stars have photometric distances estimations (van der Hucht 2001). Recently, a correlation between equivalent width of interstellar Ca IIH (3968 A) and K (3933 A) lines and Hipparcos parallaxes has been found for OB stars (Megier et al. 2005). These relations are given with formulas: $\pi=1 /[2.78 E W(K)+95]$ and $\pi=1 /[4.58 E W(H)+102]$, where $\pi$ is a parallax in arcseconds and $E W$ is the equivalent width in milliangstroms. We have used these correlations to estimate distance to Wolf-Rayet star WR 134. Equivalent widths of Ca II lines have been measured in low resolution spectra with $R=2000$. We have got: $1.17 \mathrm{kpc}$ from the Ca II H, and $1.18 \mathrm{kpc}$ the CaII K line. The distance to this star as cited in the VIIth Catalogue of Galactic Wolf-Rayet Stars (van der Hucht 2001) is $1.74 \mathrm{kpc}$. If WR 134 belongs to the complex of 
associations around Cyg OB1, then its distance agrees very well with distances of other members of these associations - also obtained from Ca II line intensities.

\subsection{SpectroWeb: an interactive graphical database of digital stellar spectral atlases}

Alex Lobel: SpectroWeb is an online maintained interactive graphical database of digital spectral atlases of bright stars at <http://spectra.freeshell.org $>$. It is an efficient and user-friendly research tool for accurate analyses of stellar spectra observed with high-spectral resolution, including the solar spectrum. The web-interface displays observed and theoretical stellar spectra, and comprehensively provides detailed atomic and molecular line information via user interaction. It fully integrates interactive spectrum visualization tools for the analysis, management, and maintenance of large volumes of spectral line-identification, -transition, and -property data. SpectroWeb 1.0 currently offers optical (3300 - $6800 \AA$ ) flux normalized high-resolution spectra of Betelgeuse (M2), Arcturus (K1), the Sun (G2), Beta Aqr (G0), Procyon (F5), and Canopus (F0). The provided line identifications are based on state of the art spectrum synthesis calculations. The graphical database is under permanent development as an online repository of identified (absorption) lines in bright star reference spectra, covering a broad range of stellar spectral types. Its object-oriented (Java) implementation offers future expansion capabilities to link and read stellar spectral atlases from various public internet sites.

\subsection{Three dimensional radiative transfer in winds of massive stars: Wind3D}

Alex Lobel, and Ronny Blomme: We discuss the development of the new radiative transfer code Wind3D. It solves the non-LTE radiative transport problem in moving stellar atmosphere models in three geometric dimensions. The code accepts arbitrary 3D velocity fields in Cartesian geometry without assumptions of axial symmetry. Wind3D is currently implemented as a fully parallelized (exact) accelerated lambda iteration scheme with a two level atom formulation. The numerical transfer scheme is efficient and sufficiently accurate to trace small variations of local velocity gradients on line opacity in strongly scattering dominated extended stellar winds. We investigate the detailed formation of P-Cygni line profiles observed in ultraviolet spectra of massive stars. We compute the detailed shape of these resonance lines to model local enhancements of line opacity that can for instance be caused by clumping in supersonically expanding winds. Wind3D is applied to preliminary hydrodynamic disk models to investigate physical properties of discrete absorption line components.

\subsection{White Dwarfs in the GALEX survey}

Adela Kawka, and Stephane Vennes: We have cross-correlated the 2dF QSO Redshift Survey (2QZ) white dwarf catalog with the GALEX 2nd data release and the Sloan Digital Sky Survey (SDSS) data release 4 to obtain ultraviolet photometry (FUV, NUV) for approximately 700 objects and optical photometry (ugriz) for approximately 800 objects. We have compared the optical-ultraviolet colours to synthetic white dwarf colours to obtain temperature estimates for approximately 250 of these objects. These white dwarfs have effective temperatures ranging from $10000 \mathrm{~K}$ (cooling age of about $1 \mathrm{Gyr}$ ) up to about $40000 \mathrm{~K}$ (cooling age of about $3 \mathrm{Myr}$ ), with a few that have even higher temperatures. We have found that to distinguish white dwarfs from other stellar luminosity classes both optical and ultraviolet colours are necessary, in particular for the hotter objects where there is contamination from B- and O-type main-sequence stars. Using this sample of white dwarfs we will determine the fraction of helium-rich white dwarfs (DO, DB) relative to hydrogen-rich white dwarfs (DA), and make luminosity functions for both hot DA and DO white dwarfs. The temperatures of the $2 \mathrm{QZ}$ white dwarfs 
obtained from the UV colours will be used to obtain luminosities of the objects and hence their distance. From this we will be able to determine the scale height of these stars.

\subsection{Relative strengths of Raman scattered He II 6545 and $H \alpha$ wings in the symbiotic star V1016 Cyg and the young planetary nebulae IC 5117}

Hee-Won Lee, and Suna Kang: Many symbiotic stars and some young planetary nebulae are known to exhibit very prominent and broad wings around $\mathrm{H} \alpha$ emission. Lee (2000) proposed that these $\mathrm{H} \alpha$ wings are formed from Raman scattering by atomic hydrogen of far UV radiation around Ly $\beta$. However, broad wings may also arise from other mechanisms including fast gas flows around the hot star and Thomson scattering. Furthermore, it is unclear whether continuum around $\operatorname{Ly} \beta$ or $\operatorname{Ly} \beta$ line photons themselves are responsible for the broad $\mathrm{H} \alpha$ wings in the proposal of Lee (2000). In order to confirm the Raman scattering origin of $\mathrm{H} \alpha$ wings, we present our high resolution spectrum around $\mathrm{H} \alpha$ of the symbiotic star V1016 Cyg and the young planetary nebula IC 5117 obtained with the Bohyunsan Optical Echelle Spectrograph installed on the $1.8 \mathrm{~m}$ telescope. The two objects show relatively strong Raman scattered He II 6545 features but H $\alpha$ wings are significantly weak in IC 5117 compared with those in V1016 Cyg. In the spectra, we note that the $\mathrm{H} \alpha$ and He II emission lines in IC 5117 are much narrower than the counterparts in V1016 Cyg. Applying Gaussian fits to these emission lines and inferring the far UV radiation compatible with the recombination theory, we perform Monte Carlo simulations of Raman scattered He II 6545 and $\mathrm{H} \alpha$ wings in an assumed neutral region with $\mathrm{H}$ I column density of $1022 \mathrm{~cm}^{-2}$. Our numerical results show relatively weak $\mathrm{H} \alpha$ wings compared with He II 6545 in IC 5117 and quite strong $\mathrm{H} \alpha$ wings in V1016 Cyg, which is consistent with our observational spectra. This result strongly indicates that the broad $\mathrm{H} \alpha$ wings are originated from the $\mathrm{Ly} \beta$ line photons, not continuum photons around $\mathrm{Ly} \beta$.

\subsection{A flare-induced mass transfer/accretion event in AM Her?}

Steven H. Saar, Vinay L. Kashyap, and Frederick A. Ringwald: We report observations of a mass transfer/accretion event in AM Her which appears to have been induced by a strong flare on the secondary. UV observations of the magnetic CV star AMHer were made with HST-sTis towards the end of a deep photometric minimum in late 2003. Our goal was to search for evidence of magnetic activity on the secondary (as seen in the Doppler-shifted hot emission lines of C IV and Si IV), one of the fastest rotating cool stars ever studied in the UV. There was little evidence for quiescent C IV emission at the velocity of the $\mathrm{M}$ star secondary, placing useful limits on its steady transition region emission. One strong flare was seen in C IV (and initially, in Si IV and He II as well), with a velocity consistent with the M-type dwarf. This was accompanied by a near-simultaneous increase in continuum emission from the white dwarf; the excess had a temperature of about $100000 \mathrm{~K}$. We argue that this strong flare may have ejected mass in the form of CME, or disturbed a large prominence system, exciting an accretion event on the white dwarf. We discuss implications of the results for low-state CV activity, mass transfer, and cool star activity at extreme rotation rates.

\subsection{White Dwarfs with strange matter cores: an analysis of candidates}

Grant J. Mathews, Nguyen Quynh Lan, In-Saeng Suh, Wolfgang Zech, Kaori Otsuki, and Fridolin Weber: We summarize masses and radii for a number of white dwarfs as deduced from a combination of proper motion studies, Hipparcos parallax distances, effective temperatures, and binary or spectroscopic masses. We construct a projection of white-dwarf radii for fixed effective mass and conclude that there is at least 
marginal evidence for bimodality in the radius distribution for white dwarfs. Some stars appear to have radii which are significantly smaller than that expected for a standard electron-degenerate white-dwarf equations of state. We argue that if such compact white dwarfs exist it is unlikely that they contain an iron core. We show that the data exhibit several features consistent with the expected mass-radius relation of strange dwarfs. We identify eight nearby white dwarfs which are possible candidates for strange matter cores and suggest observational tests of this hypothesis.

\subsection{New powerful diagnostics for hot evolved stars: constraining the hottest temperatures, the faintest winds and the neon abundance}

Luciana Bianchi, and James Herald: We have recently identified a strong P Cygni feature $(\lambda 973 \AA)$ in the far-UV spectra of some very hot $\left(T_{\text {eff }}>85000 \mathrm{~K}\right)$ central stars of planetary nebulae (CSPN) as originating from Ne VII. By including highly ionized neon calculations in stellar atmosphere models, we could reproduce this feature as it is observed in hydrogen-deficient CSPN, as well as in PG 1159 objects. The discovery of highly ionized neon diagnostic features in CSPN spectra is important because an overabundance of this element is indicative of processed material that has been dredged up to the surface as predicted in the 'born-again' scenario, a possible explanation for hydrogen-deficient CSPN. We show the potential of this strong feature as well as weaker neon features as diagnostics in stellar atmosphere analyses for extremely hot post-ABG objects (where other diagnostics are scarce). This diagnostic is potentially useful also in the analysis of massive stars with winds such as WO stars.

\subsection{A revised temperature scale for massive stars}

Luciana Bianchi, James Herald, and M. Antonieta Garcia: A consistent analysis, using non-LTE codes with sphericity and mass loss, of different ionization species in O-type star spectra observed from the far-UV to UV allowed us to constrain the long-time uncertain ionization equilibrium in the stellar winds, to quantify the role of soft X-rays from shocks and to reconcile otherwise discrepant UV line diagnostics. The modelling initially performed with the WM-basic code led to a revised (downwards) $T_{\text {eff }}$ scale for Galactic O-type stars, a result with important implications for the energy-balance calculations in H II regions and for massive star evolution. A further analysis using the CMFGEN code, including clumping effects, allowed us to resolve the remaining UV line discrepancies, in particular the $\mathrm{P} v$ line in the far-UV, and to finally reconcile mass loss and photospheric parameters derived from optical and UV lines.

\subsection{Classification and properties of Milky Way UV sources from the GALEX surveys}

Luciana Bianchi, et al.: We used Galaxy Evolution Explorer (GALEX) imaging survey data matched to the Sloan Digital Sky Survey (SDSS) in the overlapping areas to explore the nature of the UV sources. We present statistical properties of the GALEX/SDSS matched catalogs and we classify the stellar sources by comparing their seven bands photometry ( GALEX far-UV, near-UV, SDSS $u, g, r, i, z)$ to model colours computed for different astrophysical objects, taking nto account the effects of extinction. The present sample covers mostly high galactic latitudes. The GALEX photometric surveys detect hot White Dwarfs all the way throughout the MW halo, providing an unprecedentedly unbiased and possibly complete census of this elusive component of the MW. The results are compared to MW model predictions. 


\section{Updates on current UV facilities}

\subsection{Hubble Space Telescope}

Martin A. Barstow (University of Leicester): The Hubble Space Telescope has had a remarkable existence. Through the ability to service it in space, it has survived what would otherwise have been a fatal flaw in it telescope figure and its instrumentation has been successively enhanced with new technologies to keep it at the forefront of astronomical research in much the same way as a ground-based facility. For UV astronomy, it has become the work-horse facility, particularly since the termination of IUE, with the HSTFOS and HST-GHRS spectrographs initially followed by HST-STIS. Direct UV imaging has been provided by the series of HST-wide Field Cameras and, latterly, HST-ACS.

Nevertheless, space is a harsh environment. Spacecraft support systems such as gyros fail from time to time and the observatory is currently operating in a '2-gyro' mode (compared to the normal 3-gyro operations) to preserve spacecraft life time by shutting down one working gyro and saving it 'for a rainy day'. In addition to instrument changes, important subsystems have been replaced during servicing missions. At the moment, Hubble is probably instrumentally at its lowest ebb since launch. Anything other than very low resolution UV/optical spectroscopy came to an end when sTis failed in August 2004, leaving only imaging or grism spectroscopy available in the UV. Just two years later in summer 2006, the prime HST instrument ACS side-1 electronics failed. Fortunately, recovery was swift and the camera is now operating on the back-up side- 2 electronics. However, as second failure, as in the case of sTIS, would render the ACs unusable.

Following the tragic loss of Columbia in 2003, a further servicing mission to Hubble seemed very unlikely. Indeed, it was formally cancelled in 2004. However, with a successful return-to-flight of the Space Shuttle, the SM-4 mission is now back on the agenda and the go ahead was formally announced on 31 October 2006, following the successful prerequisite of two problem free shuttle missions. The SM-4 mission is expected no earlier than January 2008, but planning for this had to begin in 2005 to ensure readiness by then. The exact plans and priorities are still to be decided, but full range of activities includes:

- Replacement of failed gyros

- Replacement of batteries

- Removal of WFC2 and installation of WFC3

- Installation of COS

- Replacement of a Fine Guidance sensor

- Repair of sTis electronics

Completion of all these tasks will be very challenging and, in particular, the STIS repair will be very difficult. Furthermore, the servicing mission depends on a continuing problem-free record of Shuttle flights, comprising $\sim 6-7$ before SM- 4 . In addition, it will be necessary to have a second Space Shuttle ready for launch in case of problems with the servicing vehicle, since the latter cannot dock with the International Space Station from HSTs orbit. Nevertheless, a successful SM-4 will give Hubble a completely new lease of life, taking it well into its 3rd decade in space.

\subsection{Far Ultraviolet Spectroscopic Explorer}

Luciana Bianchi (Johns Hopkins University): Launched in 1999, the Far Ultraviolet Spectroscopic Explorer (FUSE) has extended the available ultraviolet spectroscopic range from HST's cutoff at $1150 \AA$ down to the Lyman limit at $912 \AA$. The satellite (Moos et al. 2000; Sahnow et al. 2000) consists of four co-aligned telescopes/spectrographs mounted 
on a three axis stabilized spacecraft with arcsecond pointing capability. It provides spectroscopic data in the wavelength range $905-1187 \AA$ at a spectral resolution of $R \approx 20000$. FUSE is operated from the Department of Physics and Astronomy of the Johns Hopkins University, and the mission has been extended through 2008.

Science highlights from FUSE include the discovery of an extended, tenuous halo of very hot gas surrounding the Milky Way and evidence of similar hot gas haloes around other galaxies; detection of molecular hydrogen in the atmosphere of the planet Mars; detection of molecular nitrogen in dense interstellar gas and dust clouds, at levels well below what astronomers had expected, prompting revision for theories of interstellar chemistry; and the discovery of highly ionized neon in extremely hot central stars of planetary nebulae (see also Bianchi \& Herald, this JD04, section 9.19) which has great implications for the yield of chemical elements and late evolutionary phases. Lines in the FUSE range also enabled scientists to solve long-standing uncertainties and inconsistencies in modeling atmospheres and winds from hot massive stars, which lead to a revision of effective temperatures and luminosities for O-type stars.

\section{3. $G A L E X$}

Luciana Bianchi (Johns Hopkins University): The Galaxy Evolution Explorer (GALEX) is a NASA Small Explorer mission launched in April 2003, performing imaging and spectroscopic surveys of the sky in two UV bands: near-UV and far-UV, at a depth comparable to the existing or on-going deepest surveys at other wavelengths. With a large field of view and great sensitivity, GALEX is filling a crucial gap in the spectrum, so that our knowledge of the sky from $100 \mu \mathrm{m}$ to $10 \AA$ is complete.

GALEX is surveying the sky simultaneously in two bands, far ultraviolet (FUV, 1344$1786 \AA, \lambda_{\text {eff }} \simeq 1516 \AA$ ), and near ultraviolet (NUV, $1771-2831 \AA, \lambda_{\text {eff }} \simeq 2267 \AA$ ). GALEX consists of a Ritchey-Chretien telescope with a $50-\mathrm{cm}$ aperture. It uses a dichroic beam splitter, large-format detectors, and a novel optical design to provide a circular field of view of 1.2 degrees. Observations are performed during 25 minute orbital nights (one eclipse per 98 minute orbit, the basic GALEX observational unit) outside the South Atlantic Anomaly. In imaging mode, GALEX obtains a resolution of $\sim 5 \operatorname{arcsec}$ FWHM, with 5 s photon-limited sensitivity for point sources of $m_{\mathrm{AB}} \simeq 20.5 \mathrm{in} 100 \mathrm{~s}$. In grism mode, spectral resolution $R \simeq 100-250$ is obtained for most objects in the field of view. The grism is rotated at different position angles to mitigate effects of spectrum overlap. Timetagged single photon data are stored on board and telemetered to the ground during 3 or 4 daily ground station contacts. All pointings are stationary except for a 1 arcminute spiral dither pattern to uniformly distribute detector fatigue. Night sky backgrounds are extremely low: the FUV[NUV] is dominated by diffuse galactic light [zodiacal light], with typical levels $27.5[26.5] \mathrm{mag}_{\operatorname{arcsec}^{-2}}$, corresponding to 3[30] photon/PSF in one orbital night. Astrometric accuracy is $<1$ arcsec ( $\mathrm{rms}$ ).

GALEX is providing an unprecedented, statistically powerful database of UV images and spectra of nearby and distant galaxies, and Milky Way objects (several million objects), linked to a multi-wavelength archive. GALEX has recently delivered its second major data release (GR2), which constitutes $36 \%$ of the final baseline mission data set. The GALEX data are hosted by MAST (http://galex.stsci.edu/). GR2 includes 7200 deg $^{2}$ of sky from nearly 4 million seconds of observing time.

GALEX is performing a series of imaging and spectroscopic surveys in the UV band $(1300-3000 \AA)$ that map the history, and probe the causes, of star formation over the $0<z<2$ range, $80 \%$ of the life of the universe, a period of dramatic evolution when most stars, elements, and galaxies were formed. The baseline mission focused mainly on two 
questions: (1) how does the UV trace global star formation?; and (2) what is the star formation rate (SFR) in galaxies, and how does it evolve over $0<z<2$ ?

With projected completion of the baseline mission by the end of FY07, GALEX is well on its way to fulfilling Goals 1 and 2. The proposed Extended Mission, starting in FY08, will address two other, more complex questions: (3) what are the physical drivers of star formation and its evolutionary history in galaxies?; and (4) what is the nature of the static and time-variable UV universe?

Several additional science questions are being addressed by the science team, and by the scientific community through GI programs. The extensive GALEX science data archive will allow many more investigations by the scientific community, and will provide a road map to other space-based and ground-based observing programs.

(a) The goal of the all-sky Imaging Survey (AIS) is to survey the sky to a sensitivity of $m_{\mathrm{AB}}=20.5(\sim 100 \mathrm{~s}$ exposure time $)$, comparable to the SDSS spectroscopic $\left(m_{\mathrm{AB}}=17.6\right)$ and POSS II $\left(m_{\mathrm{AB}}=21\right)$ limits. GALEX has surveyed $>13000 \mathrm{deg}^{2}$ to date, and completion of $28000 \mathrm{deg}^{2}$ is planned by the end of 2007 .

(b) The Medium Imaging Survey (MIS) has covered $1000 \mathrm{deg}^{2}$ with single-orbit $(\sim 1500 \mathrm{~s})$ exposures $\left(m_{\mathrm{AB}}<23.5\right)$, overlapping SDSS, $2 \mathrm{dF}, R O S A T$, and FIRST surveys. The MIS is $\sim 85 \%$ complete.

(c) The Deep Imaging Survey (DIS) consists of 20-orbit (30 ks, $m_{\mathrm{AB}} \simeq 25$ ) exposures, over $80 \mathrm{deg}^{2}$, of regions where major multi-wavelength efforts are already underway. 25 observations of the DIS are $~ 80 \%$ complete.

(d) The Nearby Galaxy Survey (NGS) targets well-resolved nearby galaxies for 1 2 orbits. Surface brightness limits are $m_{\mathrm{AB}} \simeq 27.5 \mathrm{deg}^{-2}$, or a star formation rate of $\sim 10^{-3} \mathrm{M}_{\odot} \mathrm{yr}^{-1} \mathrm{kpc}^{-2}$. Targets included most galaxies from the Spitzer IR Nearby Galaxy Survey, and many other surveys. The NGS is nearly complete (few hundreds galaxies)

(e) Spectroscopic surveys. Include a suite of three nested surveys

Some of the proposed surveys for the extended mission are:

(f) Galactic Cap Survey (GCS) Survey [8000 orbits]: to increase the survey at MIS depths by an order of magnitude in area over the SDSS N and S galactic caps. (see Bianchi, section 9.21 in this JD04)

(g) SDSS Deep Survey (SDS) [2000 orbits]: to obtain deep images (15000 s)over $100 \mathrm{deg}^{2}$ of the SDSS survey. This will permit a definitive study of the UV upturn and residual star formation in elliptical galaxies, star formation and chemical evolution of very low mass galaxies, and the properties of extremely red (extincted) star forming galaxies.

(h) Legacy Spectroscopy Project (LSP) [2000 orbits]: in fields that already have publicly available optical spectroscopy (SDSS), extending the coverage to $25 \mathrm{deg}^{2}$ (factor of 6 increase in SDSS overlap).

(i) Time Domain Survey (TDS) designed to exploit the excellent GALEX UV sensitivity, dark UV sky, wide field-of-view, photon-counting, repeated visits, and the contrast sensitivity of the UV emission from most variable phenomena. It will also provide a Ultra-Deep Imaging Survey (UDIS), [2000 dedicated orbits (not in other surveys); 6000 orbits total].

\section{Future UV instrumentation}

\subsection{World Space Observatory/UV}

Ana I. Gómez de Castro (UCM), Boris Shustov (INASAN), and the WSO Team: The World Space Observatory $/ U V(W S O / U V)$ is a $170 \mathrm{~cm}$ telescope equipped with three UV spectrometers covering the spectral band from Lyman- $\alpha$ (Ly $\alpha$ ) to the 
atmospheric cut-off with $R \simeq 55000$ and offering longslit capability over the same band with $R \simeq 3000$. In addition, a number of UV and optical imagers view adjacent fields to that sampled by the spectrometers. The imaging performance compares well with that of HST-ACS while the spectral capabilities are comparable to the HST-STIS echelle modes. However, with a smaller number of instruments in the focal plane, compared to $H S T$, the required number of optical elements in each subsystem is reduced. Hence, the WSO delivers considerably enhanced effective area. The planned instrument sensitivity will exceed that of HST-STIS by a factor of $10-20$. From a scientific point of view, this more than an order of magnitude improvement in UV capability will allow significant opportunities in three general directions:

(a) observe objects 4-5 magnitudes fainter than possible with HST, providing completely new opportunities in extragalactic astronomy and cosmology;

(b) carry out large scale, high resolution spectroscopic surveys of galactic sources;

(c) map the evolution of dissipative phenomena.

The WSO/UV project is led by ROSCOSMOS and it is being carried out by a broad international colaboration with participating countries: Russia, Ukraina, Germany, Italy, Spain, China, United Kingdom, Israel, France, the Netherlands, México, South Africa, Baltic Nordic Countries and Argentina (<www. inasan.rssi.ru/rus/wso/index.html>).

\subsection{Tel Aviv University UltraViolet EXplorer}

Noah Brosch (Wise Observatory): TAUVEX is the acronym for the Tel Aviv University UltraViolet EXplorer, a space telescope array designed and constructed in Israel by El-Op, Electro-Optical Industries, Ltd (a division of Elbit systems), for the exploration of the ultraviolet (UV) sky. TAUVEX was selected in 1988 by the Israel Space Agency (ISA) as its first priority scientific payload. Although originally slated to fly on a national Israeli satellite of the Ofeq series, TAUVEX was shifted to fly as part of the Spectrum Roentgen-Gamma (SRG) international observatory, a collaboration of a large number of countries with the Soviet Union (Space Research Institute IKI) leading.

Due to repeated delays of the SRG project, ISA decided in 2001 to shift TAUVEX to a different satellite. In early-2004 ISA signed an agreement with the Indian Space Research Organization (ISRO) to launch TAUVEX on board the Indian technology demonstrator satellite GSAT-4. TAUVEX is a scientific collaboration between Tel Aviv University and the Indian Institute of Astrophysics in Bangalore. Its Principal Investigators are Noah Brosch at Tel Aviv University and Jayant Murthy at the Indian Institute of Astrophysics. TAUVEX is slated to be launched in the second half of 2007.

TAUVEX consists of three bore-sighted $20 \mathrm{~cm}$ diameter telescopes on a single bezel. Each telescope images the same sky area of 0.9 degree, with an angular resolution of $7-11$ arcseconds. The imaging is onto position-sensitive detectors (CsTe cathodes on calcium fluoride windows) equipped with multi-channel plate electron intensifiers. The detectors oversample the point-spread-function by a factor of approximately 3 . The output is detected by position-sensitive anodes (wedge-and-strip) and is digitized to $12 \mathrm{bits}$. The full image of each telescope has about 300 resolution elements across its diameter.

The CsTe cathode assures sensitivity from longward of Lyman- $\alpha$ to the atmospheric limit with a peak quantum efficiency of approximately $10 \%$. The operating spectral range is separated in a number of segments selectable with filters. Each telescope is equipped with a four-position filter wheel. Each wheel contains one blocked position (shutter) and three band-selection filters.

TAUVEX is mounted to the spacecraft on a plate that can rotate around its axis (the MDP), enabling to point the telescopes' line-of-sight to any desired declination. Being on a geostationary satellite, the observation is therefore of a scanning type. A 'ribbon' 
of a constant declination, 0.9 degree wide, is scanned as time advances, completing an entire 360 degree circuit during one sidereal day. The dwell time of a source within the detector field of view is a function of the pointing declination and of the exact location in the FOV relative to the detector diameter. The closer a source is to one of the celestial poles, the longer it will reside in the TAUVEX field of view during a single scan. The longest theoretically-possible exposure is for sources at $\delta>89^{\circ} 30^{\prime}$; these can be observed for $86.4 \mathrm{ksec}$ during one day.

Each photon event hitting the detectors is transmitted to the ground in real time and processed in a near-real-time pipeline. In-between the photon events a time tag is added every $128 \mathrm{~ms}$. The time between the adjacent time tags is sufficiently short so that the orbital motion of the nadir-pointing platform is much smaller than the TAUVEX virtual pixel. Given that TAUVEX operates from a geo-synchronous platform, TAUVEX enjoys a dedicated $1 \mathrm{Mbit} / \mathrm{s}$ downlink to the ISRO control station near Bangalore. Command sequences will be uplinked after being generated by IIA and ISRO and the downlink will be analyzed on-line to monitor the payload state of health.

In most situations, TAUVEX will be able to download all the detected photon events. However, in case of strong straylight or of many bright sources in the field of view, the collected event rate may overload the capacity of the telemetry link. In this case, TAUVEX stores the photon events in a solid state memory module (4 GByte), from which the events are transmitted at the nominal $1 \mathrm{Mbit} / \mathrm{s}$ rate.

The science of TAUVEX is based on its unique characteristics: three bore-sighted and independent telescopes able to operate independently, with different filters but measuring the same sources, and reasonably fine time resolution since every detected photon is timetagged.

\subsection{Ultraviolet Imaging Telescopes}

Jayant Murthy (IIA): The Ultraviolet Imaging Telescopes are a set of two telescopes which will simultaneously image the sky in three bands from the FUV to the visible. Both telescopes are essentially identical Ritchey-Chretian designs with $38 \mathrm{~cm}$ primary mirrors. A dichroic splits the beam in one of the telescopes onto a NUV detector (RbTe photocathode) and a VIS detector (S20 photocathode) with the other telescope dedicated to the FUV (CsI photocathode). With a FOV of $0.5 \mathrm{deg}$ and a spatial resolution of better than $1 . " 5$, UVIT is intended to observe relatively large areas of the sky with a resolution comparable to ground-based observatories.

UVIT is an international collaboration led by the Indian Institute of Astrophysics (IIA). The detectors (MCP + CMOS readout) are being fabricated by Photek (UK) and integrated with photon-counting electronics by Routes (Canada) under the direction of John Hutchings and the Canadian Space Agency; the optics are fabricated by LEOS, a division of ISRO; and IIA is responsible for the structure and the instrument integration. A full calibration of the detectors (only) will be done in Canada with the focusing, characterization and integration done at new facilities built at IIA.

UVIT will launch as part of the ASTROSAT satellite which includes several X-ray telescopes (see <http://www.rri.res.in/astrosat> for more information). ASTROSAT is India's first satellite dedicated to astronomy and is scheduled for launch in late 2008. The mission is driven by proposals with time shared between the different collaborators with some part reserved for international proposers. The data will be archived at and disseminated from the Indian Space Science Data Centre located in Bangalore. After an initial proprietary period, the data will be released to the astronomical community.

UVIT is intended to be the beginning of an active thrust into UV astronomy at IIA. A significant investment is being made into an astronomical instrumentation program with 
further space programs under planning. An integration and calibration facility is almost complete at which future space payloads can be developed and made ready for flight.

\subsection{Stellar Imager}

Kenneth G. Carpenter (NASA GSFC), Carolus J. Schrijver (LMATC), Margarita Karovska (SAO), and the SI Vision Mission Study Team: The Stellar Imager (SI) is a UV-optical, space-based interferometer designed to enable 0.1 milliarcsec spectral imaging of stellar surfaces, and of their interiors via asteroseismology, and of the Universe in general. SI is identified as a "Flagship and Landmark Discovery Mission" in the 2005 NASA Sun Solar System Connection (SSSC) Roadmap and as a candidate for a "Pathways to Life Observatory" in the NASA Exploration of the Universe Division (EUD) Roadmap (May, 2005). The ultra-sharp images of the Stellar Imager (<http://hires.gsfc.nasa.gov/si/>) will revolutionize our view of many dynamic astrophysical processes. The 0.1 mas resolution of this deep-space telescope will transform point sources into extended sources, and snapshots into evolving views. SI's science focuses on the role of magnetism in the Universe, particularly on magnetic activity in stars like the Sun. SI's prime goal is to enable long-term forecasting of solar activity and the space weather that it drives in support of the Living With a Star program in the Exploration Era. SI will also revolutionize our understanding of the formation of planetary systems, of the habitability and climatology of distant planets, and of many magneto-hydrodynamically controlled processes in the Universe. Concept development for the full-up mission and for a possible smaller "pathfinder" mission continues and needed technology development, driven by $S I$ and numerous other missions (SPECS, $B H I / M A X I M, L F, P I)$, proceeds in the areas of precision formation flying (e.g., the Synthetic Imaging Formation Flying Testbed), a GSFC/MIT/MSFC collaboration) and closed-loop optical control of sparse arrays (e.g., the Fizeau Interferometer Testbed (FIT) at GSFC). The Pathfinder mission could be ready for launch in the next decade and the full mission by 2025, if they were selected for flight in future NASA and/or ESA opportunities.

\subsection{The Super-Earths Explorer}

Jean Schneider (LUTH - Paris Observatory), Pierre Riaud (LESIA, Paris Observatory), Giovanna Tinetti (IAP, Paris) and the SEE-COAST Team: The Super-Earths Explorer Coronagraphic Off Axis Space Telescope (SEE-COAST) is a space mission project to be submitted to ESA. It is mainly devoted to the diected imaging, in the visible and near UV (down to Ly $\alpha$ ), of extrasolar planetary companions reflecting the light of their parent star. It will also measure the polarization of planets. As a complementary programme, it will detect circumstellar disks, AGNs and investigate stellar activity.

\section{Summary of JD 4}

\subsection{Introduction}

The scientific contributions to this JD have shown the broad range of problems within stellar astrophysics that require development of new UV instrumention. There are two ways to approach this need: observatory class facilities and space missions that have a much more narrowly defined, and often single, science goal.

The observatory approach assumes that continued access to the UV-range has to be granted to the world-wide community in routine manner (similar to the access to radioinfrared or optical ranges). In this sense, multiple purpose instrumentation needs to be 
defined that suits most of the needs of the astronomical community. The World Space Observatory/UV represents one example of this approach, as described in Section 11.1. A handicap is the high cost of space facilities. There are, however, some relevant processes whose understanding requires the development of specific instrumentation, often at the edge of the technological capabilities of modern engineering. At the end of this JD04 there was a lively discussion on future UV instrumentation, which we report in Section 11.

\subsection{Observatory-class access to the $U V$-range}

At the end of 2002, a group of European astronomers coming from a broad range of disciplines: from fundamental astrophysical research to observational expertise in the optical, UV and X-ray ranges, as well as space instrument development teams, joined efforts to evaluate the need to develop new UV instrumentation for the coming decade. This group set the seed for the Network for UltraViolet Astrophysics (NUVA) to establish some of the major scientific objectives of the astronomical community and to fully exploit the large astronomical facilities planned for other spectral ranges.

At that time, HST-STIS was still working in and the Cosmic origins spectrograph (COS) was being built for replacement. HST was expected to last till 2010/12, FUSE was working nominally, and GALEX was close to launch. However, the major space agencies had no plans for new UV spectroscopic missions, apart from the large optical/UV telescope included in the NASA Origins plan, expected to enter development phase in 2015 - 2020 ready for a launch in the second quarter of this century. On Friday 16 January 2004 NASA decided that the planned Shuttle mission to service and upgrade HST (SM4), including the repair of sTIS and installation of COS, would be cancelled. Just a few months later sTIS failed. No access to high resolution UV spectroscopy was available during most of 2005, until FUSE resumed observations in November 2005 after the failure of the third of the four onboard reaction wheels in December 2004. Therefore, the need for new missions to fly during the next decade was becoming urgent. Fortunately, the earlier decision on the SM-4 (the final) Hubble servicing mission was reversed on October 31st, 2006. The complete plan covers the installation of two new instruments HST-COS and HST-wide Field Camera 3, both with UV capability. In addition, gyros and batteries will be replaced and HST-STIS will be repaired. This will lead to a new lease of life for HST as a UV observatory and should allow the mission to continue at least to its current projected end $\sim 2012$.

The NUVA (<www.ucm.es/info/nuva>) was officially established in January 2004. A detailed study of the needs for UV instrumentation in the many fields of astrophysical research can be found in a special issue of Astrophysics and Space Science, entitled Fundamental problems in astrophysics: requirements for future UV observatories (Gómez de Castro et al. 2006) which summarizes the thoughts and work of the NUVA and the UV community at-large. A list of the fields in stellar astrophysics requiring access to this range was elaborated; these are:

- Solar System: planets atmospheres, magnetospheres and interaction with the Solar wind.

- Cool Stars: Mapping dissipative processes (heating of the chromosphere and the corona), winds, evolution of solar-like dynamos, extending the knowledge to brown-dwarfs structure.

- Massive stars: wind clumpiness and DACs evolution, relation with magnetic fields, massive stars in the Local Group and further out. 
- ISM: disk halo interaction and HVCs, energy input into the ISM (mechanical bubble, supernovae; radiative), chimneys, halo heating and galactic winds. Extending this work to the Local Group.

- Star Formation-I: outflows-powering by accretion, accretion geometry, mapping dissipative processes, evolution to the main sequence, mapping dissipative processes, influence of dissipative processes in the evolution of the MRI and the inner disk.

- Planets: Evolution of young planetary disks, comets in extrasolar systems, evolution of comets-hive and its impact on the evolution of young planetary systems. Detection and characterization of the atmospheres of extrasolar planets.

- Star formation II: violent star formation processes in galaxies, the interaction between the stellar clusters and the ISM and the variation of the IMF, contribution of starbursts to the reionization of the Universe, chemical enrichment of the IGM, role of starburst on AGNs

- White dwarfs: the origin and relationships of the $\mathrm{H}$ and He-rich groups, the initialfinal mass relation for white dwarfs and their progenitors and the 3D structure of the ISM; to increase the sample of WD and extend it to the globular clusters and nearby galaxies.

- Interacting binaries: nature of SN Ia progenitors exploring both single and doubledegenerate channels, the physics of accretion discs, in particular the role of viscosity and its time-dependence, and the development of winds, the fundamental properties of white dwarfs in CVs, the nature of the IB population in globular clusters.

- Globular clusters and stellar evolution.

- Planetary nebulae.

- Supernovae: SN explosions, characterize SNIa across the Universe.

Most of science described there could be carried out with two basic instruments:

(a) A high-resolution $(R=50000-100000)$ spectrograph covering the whole $90-320 \mathrm{~nm}$ spectral range.

(b) A low-spectral resolution $(R=1000-5000)$ high-sensitivity spectrograph allowing integral field spectroscopy (long-slit in its simplest version) with spatial resolution (50 mas) and wavelength coverage from $110-450 \mathrm{~nm}$.

These instruments should provide an improvement by a factor of $\sim 20$ in effective area over the HST-STIS capabilities. Additional features demanded by the community are highresolution imaging (better than 80 mas) and an orbit that allows mapping the temporal evolution of dissipative phenomena and a rapid response to 'Targets of Opportunity'. High-time resolution is also required to map the evolution of rapid phenomena. Groups working on the atmospheres of extrasolar planets also expressed interest in some overlap with the optical range (from $3200 \AA$ to $3500 \AA$ ).

This projected improvement is somewhat conservative from the technological point-ofview, since it is mostly achieved by improving optical designs and coatings and making use of detectors with enhanced sensitivity. Larger detector formats are also available and fast read-out electronics gives improved dynamic range. It is amazing this progress that can be achieved with a relatively modest investment coupled to a modest sized telescope. A good example of this is the proposed World Space Observatory project.

However, looking into the far future, it is clear that the frontier of UV astronomy will require construction of much larger aperture facilities that increase the effective collecting surface by 2 orders of magnitude or more. A properly instrumented $4-6 \mathrm{~m}$ telescope in space will be essential for future UV observations. 


\subsection{Single science objective facilities}

This approach requires the identification of the fields of astrophysics which are the most relevant for the progress of physics. There is a general agreement on those being:

- The characterization of dark energy

- Physics in extreme environments: black holes, SNe or the polarized vacuum.

Another important field is to understand life sustainability in the Universe and among it,

- The magnetic 'stability' of the Sun and solar-like stars and its role in the evolution of life in the associated planetary systems.

- The formation of planetary systems

- The characterization of planetary systems.

All these projects require extension of the effective area of the telescopes by at least two orders of magnitude over HST-STIS (or one order of magnitude over HST-COS, WSO/UV), since most of these science goals require absorption spectroscopy of weak/distant far UV sources.

\subsection{Discussion}

The situation of the various on-going projects was described during the discussion, especially, the possible (now approved) mission to service and upgrade $H S T$ and the WSO/UV project.

Once detector and instrument systems reach their ultimate efficiency, as is now occurring, the factor of 100 increase in effective area can only be realised by increasing the collecting surface. This implies that these future projects, if feasible, are going to be very expensive and will need to be able to answer at least one of the key questions of science to justify their cost. The most realistic approach seems to be that followed by the Stellar Imager in proposing a cluster of small coordinated telescopes. Another possible option a Moon-based observatory, where a large telescope could be constructed using technologies currently applied to large ground-based telescopes.

An important issue raised during the meeting is the difficulty in gaining the support of the community at large for very focussed projects as well as the need to have teams of instrumentalists and observers working together. Concern was expressed about the diminishing number of these teams.

\section{Acknowledgements}

It is a great pleasure to acknowledge the support of the members of the IAU XXVI General Assembly LOC who dealt brilliantly with the difficult task of organizing a plethora of small and large meetings, joint discussions and discourses involving around 2500 astronomers.

\section{References}

Gómez de Castro, A. I., Wamsteker, W., Barstow, M. A., et al. 2000, ApESSS, 303, 133

van der Hucht, K. A., Schrijver, H., Stenholm, B., et al. 1997, New Astronomy, 2, 245

van der Hucht, K. A. 2001, New Astronomy Reviews, 45, 135

Moos, H. W., Cash, W. C., Cowie, L. L., et al. 2000, ApJ (Letters), 538, L1

Sahnow, D. J., Moos, H. W., Ake, T. B., et al. 2000, ApJ (Letters), 538, L7 